\title{
Environmental and financial performance evaluation of a helicopter's canopy production using different materials and manufacturing processes
}

\author{
Ch.V.Katsiropoulos ${ }^{1, *}, A$. Loukopoulos $^{1}$, and Sp.G. Pantelakis ${ }^{1}$ \\ ${ }^{1}$ Laboratory of Technology \& Strength of Materials, Dept. of Mechanical Engineering \& Aeronautics, \\ University of Patras, Panepistimioupolis Rion, 26500 Patras, Greece
}

\begin{abstract}
In the present work Life Cycle Analysis (LCA) and Life Cycle Costing models (LCC) were developed for quantifying the financial and environmental performance of different material (carbon fiber reinforced thermosetting and carbon fiber reinforced thermoplastic composites) and manufacturing scenarios (autoclave, RTM and CDF) associated with the production of aeronautical structural components. To validate the models developed, they were implemented for the case of a helicopter's canopy. The results from the analysis pointed out the environmental and financial ${ }^{1}$ advantage of producing the canopy from carbon fiber reinforced thermosetting composites involving RTM as the manufacturing process. On the other hand, the environmental and financial viability of the scenarios including thermoplastic composites as the material of choice is impaired from both the high embodied energy and raw material cost of PEEK. However, potential benefits from thermoplastic composites like recyclability and reusability as well as the high production rates that they offer and not taken into account in this study could improve their environmental and financial viability. This underlines the need to include potential reusing and recycling applications of the composites, as well as circular economy considerations to the criteria for designing an aircraft structure, selecting the material for this structure and finally manufacturing the structure.
\end{abstract}

\section{Introduction}

The selection process of the appropriate material for a particular application in aeronautics is a vital step at the early design stages and must fulfill a number of criteria (weight reduction, cost minimization, efficient mechanical properties, etc.). The mainstream material for aeronautical applications is carbon fiber reinforced thermosetting composites because of their low processing temperatures, low viscosity, superior adhesion to fibers, fatigue strength etc. However, issues associated with their long curing cycles which lead to low production rates combined with the growing environmental concerns associated with their end of life treatment, as well as the adoption of stricter environmental policies have turn the attention of the aeronautical industry to thermoplastic composites. Thermoplastic composites exhibit superior impact and chemical resistance, unlimited self-life, reduced processing time leading to higher production rates, ability of assembling sub-structures by

\footnotetext{
* Corresponding author: xkatsiro@mech.upatras.gr
} 
welding leading to a weight reduction and recyclability, properties that thermosetting composites are unable to provide. On the downside, the higher processing temperatures and pressures needed for processing these materials leading to both increased energy consumption and tooling cost are key barriers for their wide scale adoption for the construction of primary structures from the aeronautical industry. As far as the manufacturing techniques are concerned, out of autoclave techniques demonstrate significant financial and environmental benefits as compared to the conventional autoclave process however autoclaving is expected to lead to a higher product quality. These conflicting parameters underline the necessity of developing tools and concepts allowing the simultaneous optimization of a product with regard to quality, cost and environmental impact.

The aim of this work is to make a comparative analysis from an environmental and financial standpoint for different material, manufacturing and recycling scenarios associated with the production of an aeronautical structural component.

\section{Definition of the case of study}

In the present work alternative material, manufacturing and recycling scenarios are investigated in terms of environmental and financial viability for producing the canopy of the EUROCOPTER EC Twin Star helicopter described in [1], Fig.1.Two material options (carbon fiber reinforced epoxy composite (TS)/ carbon fiber reinforced PEEK composite (TP)) and three different processing routes namely the autoclave, RTM and Cold Diaphragm Forming (CDF) were considered. The autoclave and RTM accounted for both thermosetting and thermoplastic composite whereas the CDF only for the thermoplastic composite. As far as the end of life treatment of the canopy is concerned, mechanical recycling was considered for both thermoplastic and thermosetting composites whereas pyrolysis only for the thermosetting composites.

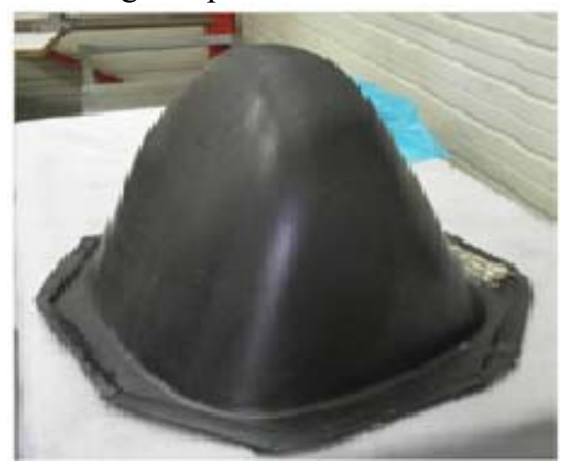

Figure 1:The canopy described in [1] (1:3 scale prototype of the real part).

\section{Life Cycle Analysis}

Life cycle analysis is a standardized technique (ISO 140402006 [2]) for assessing the environmental footprint associated with a product or a process, from raw material extraction to disposal or recycling.

Based on this concept, an LCA model was developed for evaluating the environmental footprint of the canopy of the Twin Star Helicopter. The stages taken into consideration for the present LCA were: carbon fiber production, epoxy and PEEK production, manufacturing and recycling. The processes under investigation were considered to be all 
electric. For each stage the total energy demands as well as the Global Warming Potential100 (GWP100) for a period of 100 years were estimated. The GWP100 is an index commonly used among LCA studies for evaluating the environmental footprint and is suitable for this study because electricity generation mainly produces $\mathrm{CO} 2$ emissions. The total energy consumption was calculated by multiplying the mass of each material with the energy intensity of each process (eq.1). The energy intensity of each process was derived from literature [3-7]. Since the processing temperature of PEEK is almost three times higher from the processing temperature of epoxy, the energy demands at the manufacturing stage were considered three times higher as well. The GWP100 was calculated by multiplying the $\mathrm{kg} \mathrm{CO} 2$ eq. produced from the consumption of $1 \mathrm{kWh}$ of electricity with the total energy demands of each process (eq.2). The $\mathrm{kg}$ CO2eq produced from the consumption of $1 \mathrm{kWh}$ were considered equal to $0,34 \mathrm{~kg} \mathrm{CO} 2 \mathrm{eq} / \mathrm{kWh}[8]$.

$$
\begin{array}{r}
E_{i}=m_{i} e_{i} \\
G W P_{100}=E_{i} \frac{m_{\mathrm{CO}_{2} e q}}{k W h}
\end{array}
$$

\section{Life Cycle Costing}

The Life cycle cost analysis is a suitable tool for determining financial trade-offs arising from a product or a process. In this study an LCC model was developed based on the principles of the Activity Based Costing method (ABC) for calculating the total cost and indentify the major cost drivers. $\mathrm{ABC}$ is an accounting method implementing cost estimation relationships (CERs) for expressing the cost as a function of one or more independent variables that take into account the geometrical features of a material or a product like the perimeter, surface, length, shape complexity, mass, etc. In the performed analysis, costs associated with labor, material, energy and recycling were calculated, according to the total cost relation (3). CERs were either formulated or adopted from [1]. The recycling cost was assumed equal to the energy cost of the recycling process. For the purpose of the LCC analysis the cost of $1 \mathrm{kWh}$ was considered equal to 0,114 Euros [9] and the labor cost equal to 32,6 Euros/hour [9]. The cost of the raw material was considered equal to the cost of its constituents. Additionally, the empirical assumption of $80 \%$ of scrap material based on [1] was made for all the manufacturing processes. The energy cost for all the processes was calculated as follows:

$$
\begin{array}{r}
K_{\text {total }}=K_{\text {labor }}+K_{\text {material }}+K_{\text {energy }}+K_{\text {recycling }} \\
K_{i}=E_{i} k_{i}
\end{array}
$$

where $\mathrm{Ki}$ is the total energy cost, $\mathrm{Ei}$ is the total energy consumption and $\mathrm{k}$ is the cost of 1 $\mathrm{kWh}$ of electricity. 


\section{Results and Discussion}

\subsection{Life Cycle Analysis}

From the performed environmental analysis, the production of the raw material (carbon fiber and resin) is responsible for $90 \%$ of the total environmental impact. Producing the canopy using carbon fiber reinforced epoxy composite and involving RTM as the manufacturing process demonstrates the lowest environmental footprint (figures 2,3). This is due to the lower processing temperature and embodied energy of the epoxy composite as compared to the thermoplastic (figure 4). Additionally, out of autoclave techniques were found to outperform autoclaving. On the contrary, the worst practice for producing the canopy is when carbon fiber reinforced thermoplastic composite is the material of choice and autoclaving is the manufacturing process because of the high embodied energy and processing temperature of PEEK as well as the higher energy demands of the autoclave. The environmental performance of producing a thermoplastic canopy is improved when out of autoclave techniques (RTM and CDF) are considered as the manufacturing processes but this amelioration is not enough to provide a lower life cycle impact.

However, this study investigates the environmental footprint only in terms of energy demands leading to the paradox conclusion that a non-recyclable material (epoxy composite) is a more environmentally friendly alternative as compared to a recyclable material (PEEK composite). It should be noticed that Landfill is a relatively cheap disposal route and mostly used in overseas countries (eg. USA) but is the least preferred waste management option under the European Union's Waste Framework Directive, and opposition to it is expected to increase over the coming years; it is already forbidden in Germany, and other EU countries are expected to follow this route. In this frame, many different recycling techniques have been studied and implemented for the last two decades in Europe: mechanical processes (mainly grinding), pyrolysis, solvolysis and other thermal processes [10].

This controversial result underlines the need to include potential reusing and recycling applications of the composites, as well as circular economy considerations to the criteria for designing an aircraft structure, selecting the material for this structure and finally manufacturing it.
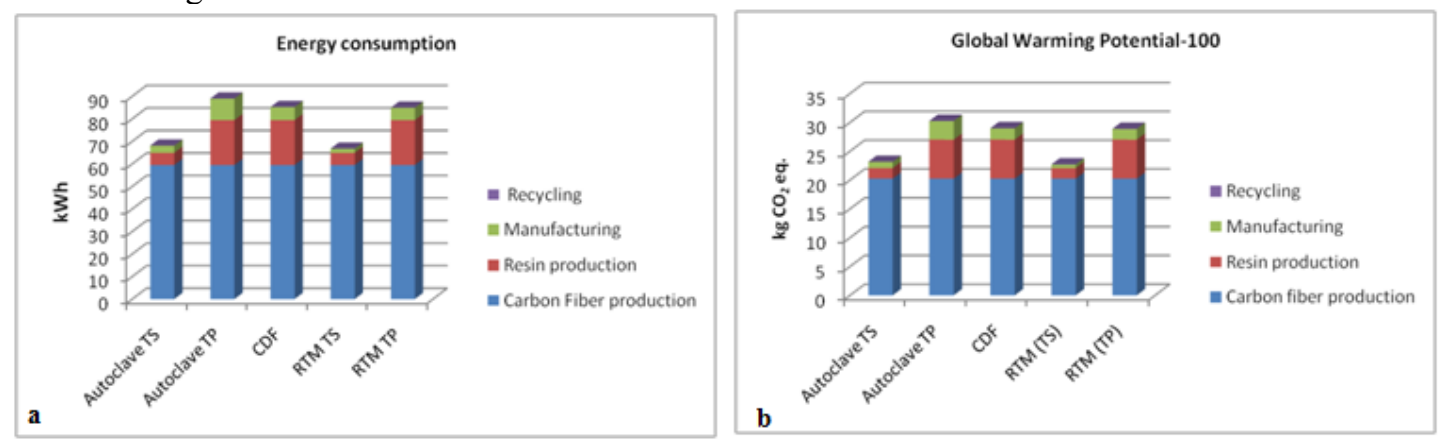

Figure 2: (a) Total energy consumption and (b) Global Warming Potential-100 for each process scenario when mechanical recycling is considered. 

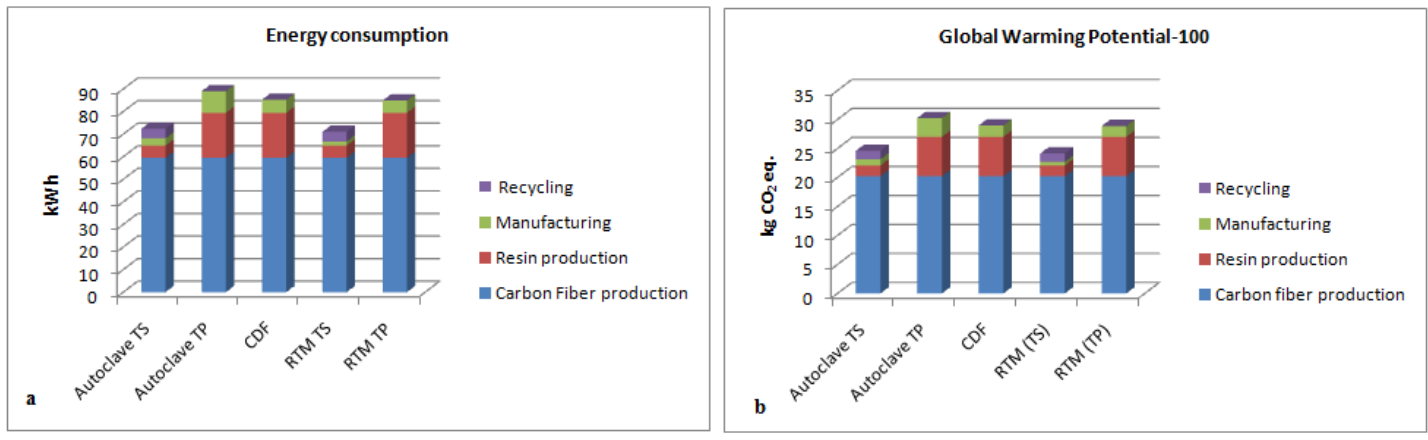

Figure 3: (a) Total energy consumption and (b) Global Warming Potential-100 for each process scenario when mechanical recycling is considered for thermoplastic and pyrolysis for thermosetting composite.

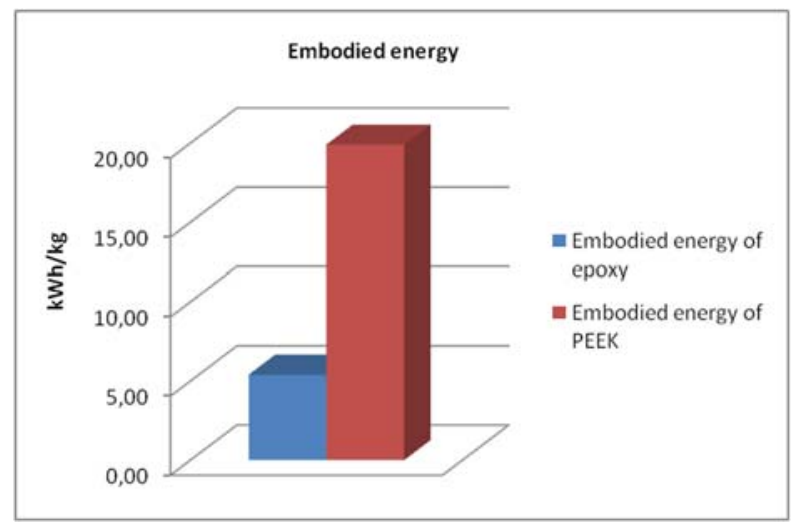

Figure 4: Embodied energy of epoxy and PEEK resin.

\subsection{Life Cycle Costing}

From figure 5 it is obvious that the main contributors to the total cost are the material and the labor costs. On the other hand, the energy and the recycling cost occupy a negligible portion of the total cost (less than 1\%). For this reason only the scenarios where mechanical recycling is considered as the recycling technique are shown. Moreover, the results from the performed analysis underlined the financial benefits occurring from producing the canopy from carbon fiber reinforced epoxy composite using the RTM technique. This is because of the lower raw material cost of the epoxy composite as compared to thermoplastic and of the absence of auxiliary materials (breather films, vacuum bags, diaphragms etc.). Furthermore, the less steps needed for preparing the mold decreases the labor cost of RTM by $35 \%$ and by $26 \%$ as compared to the conventional autoclave technique and CDF respectively (figure 6). The financial performance of the scenarios when PEEK composite is considered is impaired from the high raw material cost. However, it should be expected that the financial performance of the thermoplastic composites will be appreciably improved by considering the higher production rates offered from these materials as a decision making parameter, as well as by accounting for their high reusability potential. 
Figure 7 demonstrates a characteristic example of the effect of the main cost drivers to the total cost of an activity. It exhibits the cost variation for storing the component as a function of two product's features, the weight and the perimeter, revealing that this activity is more sensitive to a perimeter increase than to a weight increase.

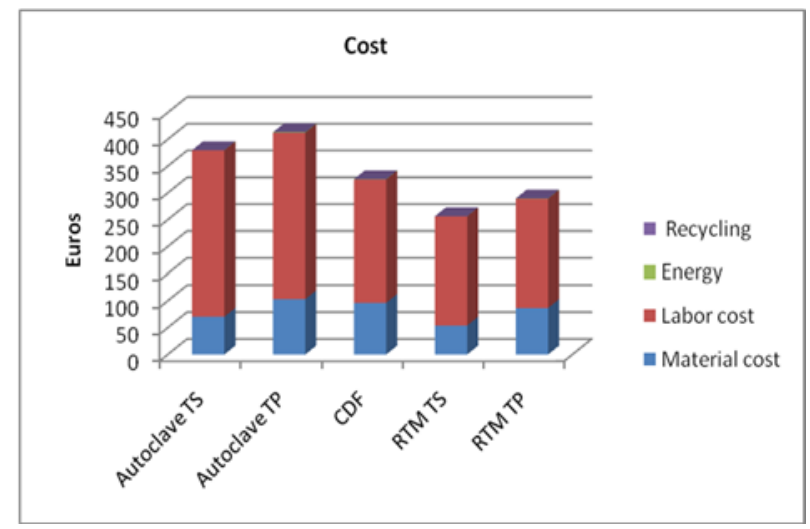

Figure 5: Total cost for the different manufacturing scenarios examined.
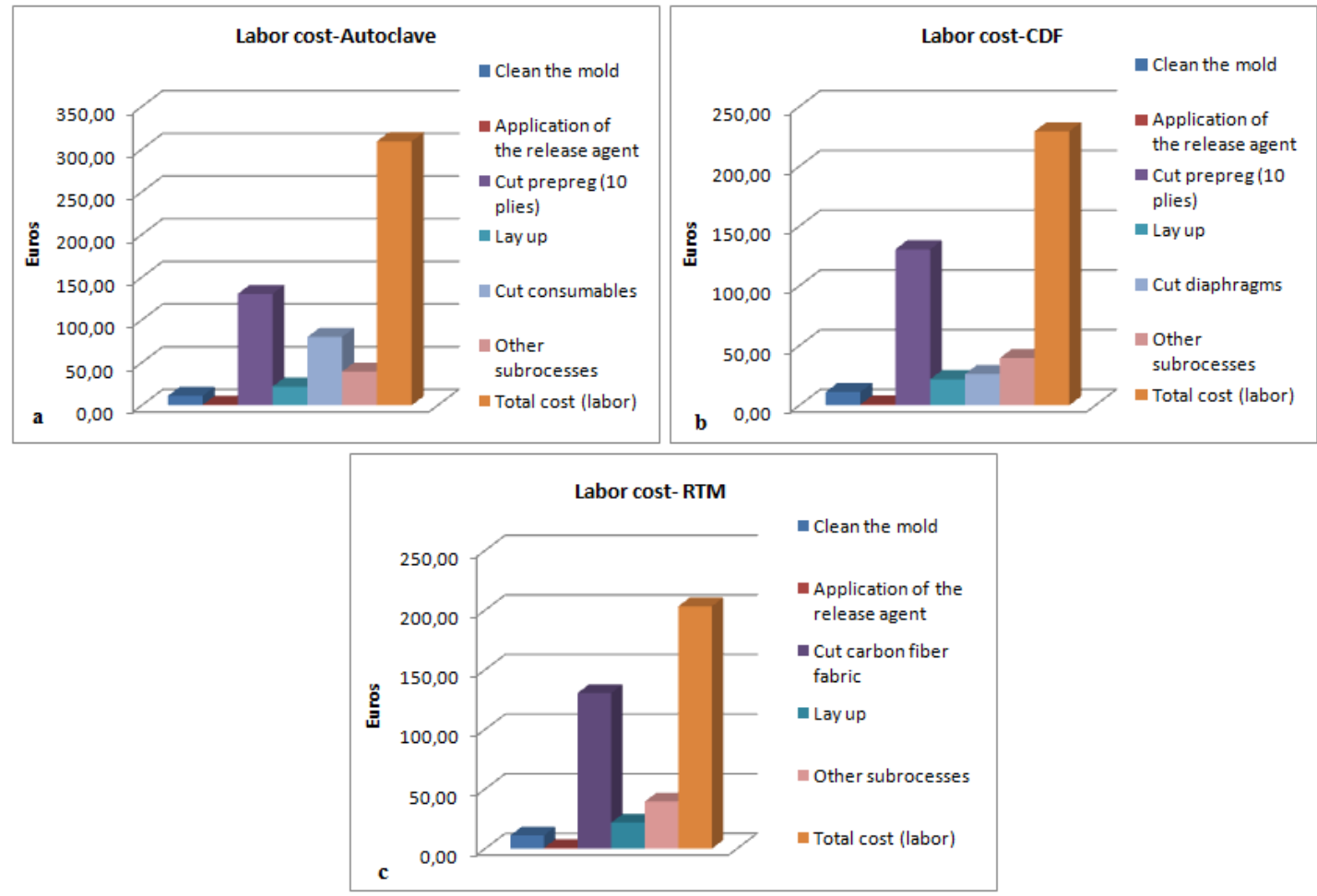

Figure 6: Labor cost breakdown structure for a) Autoclave, b) CDF, c) RTM. 


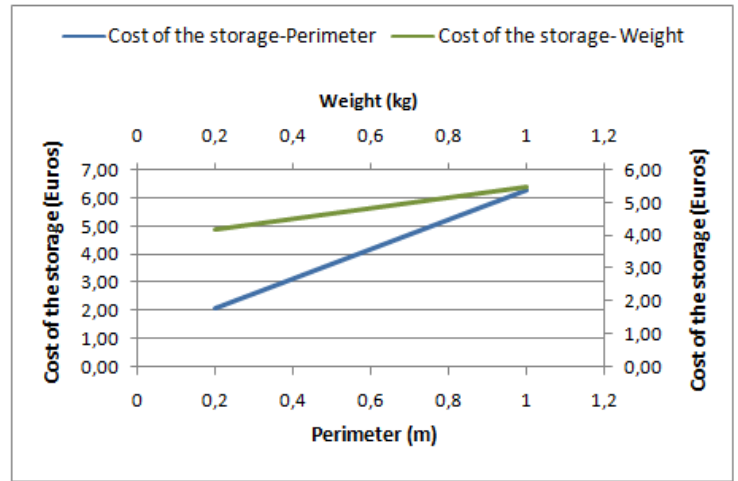

Figure 7: Cost of the storage as a function of the perimeter and of the weight.

\section{Conclusion}

In this study Life Cycle analysis and Life Cycle costing models were developed so as to quantify the environmental and financial impacts of alternative material (carbon fiber reinforced epoxy and carbon fiber reinforced PEEK composite) and manufacturing processes (autoclave RTM, and CDF) associated with the production of a helicopter's canopy. The results from the analyses demonstrated that the best practice for producing the canopy is the choice of carbon fiber reinforced composite by involving RTM as the manufacturing technique. This is because of the lower energy intensity, lower processing temperature and cost of the thermosetting composite. Additionally, RTM is a technique that requires a limited number of steps for the lay- up leading to a reduced labor cost as compared to autoclave and CDF. The high embodied energy, processing temperatures and cost of PEEK composite are responsible for the lower ecological and financial performance as compared to thermosetting. On the other hand, parameters not taken into account in current material and process selection practice, like the great potential of reusability of Thermoplastics as compared to Thermosets, their higher product quality (sufficient mechanical properties and lack of defects) that autoclaving provides, productivity (thermoplastic composites offer reduced processing cycles), assembling (the weldability of thermoplastic composites is expected to lead to a lower assembling cost and a weight reduction), can affect the decision strategy at the early design stages. The above findings underline the need for developing a versatile concept able of dealing with intricate tasks and provide the optimal design options among different alternatives.

The present work is a preliminary study performed within NHYTE project [11]. The NHYTE project has received funding from the European Union's Horizon 2020 research and innovation programme under grant agreement No 723309.

\section{References}

1. S. G. Pantelakis, C. V. Katsiropoulos, G. N. Labeas, and H. Sibois, "A concept to optimize quality and cost in thermoplastic composite components applied to the production of helicopter canopies," Compos. Part A Appl. Sci. Manuf., vol. 40, no. 5, pp. 595-606, (2009)

2. ISO 14040, (2006) 
3. E. Incorporated, "Bandwidth Study on Energy Use and Potential Energy Saving Opportunities in the Manufacturing of Lightweight Materials: Carbon Fiber Reinforced Polymer Composites," (2016)

4. T. Suzuki and J. Takahashi, "Prediction of energy intensity of carbon fiber reinforced plastics for mass-produced passenger cars," Ninth Japan Int. SAMPE Symp. JISSE-9, pp. 14-19, (2005)

5. Ashby, "Material Property Data,", (2016)

6. J. Howarth, S. S. R. Mareddy, and P. T. Mativenga, "Energy intensity and environmental analysis of mechanical recycling of carbon fibre composite," J. Clean. Prod., vol. 81, pp. 46-50, (2014)

7. S. Job, G. Leeke, P. T. Mativenga, G. Oliveux, S. Pickering, and N. A. Shuaib, "Composites recycling: Where are we now?," (2016)

8. www.electricitymap.org

9. ec.europa.eu

10. Graldine Oliveux, Luke O. Dandy, Gary A. Leeke, "Current status of recycling of fibre reinforced polymers: Review of technologies, reuse and resulting properties", Progress in Materials Science, vol. 72, (2015), pp. 61-99

11. New Hybrid Thermoplastic Composite Aerostructures manufactured by Out of Autoclave Continuous Automated Technologies (NHYTE). (EU project, 2017-2020). 